\title{
Nonoperative Management of Common Shoulder Conditions
}

\author{
Brandon Tauberg1*, Kevin Denehy², John Prodromo², Neil S. Kumar ${ }^{2}$, Mary K. Mulcahey ${ }^{2}$ \\ ${ }^{1}$ Department of Orthopaedic Surgery, Montefiore Medical Center, Bronx, NY, USA \\ ${ }^{2}$ Department of Orthopaedic Surgery, Drexel University, Philadelphia, USA
}

Received: April 24, 2015; Accepted: July 20, 2015; Published: September 18, 2015

*Corresponding author: Brandon Tauberg, MD, Department of Orthopaedic Surgery, Montefiore Medical Center, 1250 Waters Place, Tower 1 11th Floor, Bronx, NY 10461, USA, Tel: 001-347-577-4416; Fax: 001-215-762-3442; E-mail: bmt5032@gmail.com

\begin{abstract}
Shoulder pain is a common complaint encountered by physicians. There are many different etiologies of shoulder pathology, with three of the more common disease processes being rotator cuff tendinitis, biceps tendinitis, and adhesive capsulitis. Frequently, these conditions can be managed non-operatively with very good results. Non-operative treatment includes rest, anti-inflammatory medications, corticosteroid injections, and physical therapy. There are subtle differences in the treatment algorithms that can optimize clinical outcomes. It is important for the treating physician to understand these differences and to tailor the treatment plan accordingly.
\end{abstract}

Keywords: Rotator cuff; Biceps tendon; Frozen shoulder; Tendinitis; Adhesive capsulitis

\section{Introduction}

Shoulder pain is a popular complaint both in the primary care and orthopedic outpatient setting. Sixteen to thirty-four percent of the general population suffers from shoulder pain [1]. The etiology of pain can often be determined with a focused history, thorough physical examination, and appropriate imaging. Rotator cuff tendinitis, biceps tendinitis, and adhesive capsulitis are common shoulder conditions that frequently resolve with non-operative treatment. Implementation of a physical therapy program that is focused on shoulder girdle range of motion and strengthening often improves shoulder function and decreases discomfort [2]. While physical therapy is a mainstay of treatment, its outcome and effectiveness depend on the etiology of the condition [2-4]. This review will discuss the anatomy, etiology, and non-operative treatment options for rotator cuff tendinitis, biceps tendinitis, and adhesive capsulitis.

\section{Rotator Cuff Tendinitis}

Rotator cuff disorders are a common cause of shoulder pain in the general population and are associated with an impaired quality of life [1,2]. Although "tendinitis" describes acute and chronic conditions involving an inflammatory process, a minimal number of inflammatory cells are found in the rotator cuff tendons and subacromial bursa [5].Therefore, many healthcare professionals consider "rotator cuff tendinitis" to be a misnomer and feel that it is more accurately described as a "tendinosis" or "tendinopathy" [5]. Tendinopathy is defined as an overuse condition manifested by pain in or around tendons secondary to failure of tendon regeneration [6,7]. Tendinosis is defined as degeneration and disorganization of collagen with increased vascularity and cellularity without obvious inflammatory cells $[7,8]$. Neer described rotator cuff disorders as occurring on a spectrum, progressing from tendinitis to tendinosis with degeneration, partial thickness, or full thickness rotator cuff tears [9]. Throughout this section, rotator cuff tendinitis will be defined as a general term, referring to chronic tendon pain associated with overuse, irritation, strain, degeneration, and poor mechanics [7].

\section{Anatomy}

The rotator cuff is the primary dynamic stabilizer of the glenohumeral joint, compressing the humeral head within the glenoid fossa during motion, and is critical for shoulder rotation and elevation [10]. The supraspinatus abducts the forwardflexed arm, minimally contributing to external rotation, and is innervated by the suprascapular nerve [11,12]. The infraspinatus, also innervated by the suprascapular nerve, contributes mostly to external rotation of the arm, though the deltoid, teres minor, and supraspinatus assist with this action $[8,13]$. Teres minor, innervated by the axillary nerve, assists with external rotation and abduction when the arm is abducted to $90^{\circ}$, powering up to $45 \%$ of external rotation [14]. The subscapularisis innervated by the subscapular nerve and is responsible for internal rotation of the shoulder and adduction of the arm [12]. The major arterial supply to the rotator cuff is derived from the ascending branch of the anterior humeral circumflex artery, the acromial branch of the thoracoacromial artery, the suprascapular artery, and posterior humeral circumflex artery [15].

\section{Pathology}

Repetitive activities that involve overhead reaching, pushing or lifting with outstretched arms often lead to the development of rotator cuff tendinitis. The supraspinatus tendon is the most frequently injured tendon [16]. ${ }^{16}$ As mentioned previously, rotator cuff tendinitis is not a true inflammatory condition; pathology is thought to be a result of biomechanical or vascular 
factors, though it is generally agreed upon that a combination of both is most likely [17]. ${ }^{17}$ Furthermore, intrinsic and extrinsic mechanisms may contribute to the development of rotator cuff tendinitis. Intrinsic mechanisms of tendinopathy are associated with the tendon itself and include aging, altered biology, and disruption of the microvascular blood supply. Extrinsic mechanisms are compressive forces exerted by surrounding structures that increase the risk of developing impingement during physiologic range of motion of the shoulder $[17,18]$. Both intrinsic and extrinsic mechanisms cause disruption of the tendon microarchitecture, resulting in a variable response to cyclic loading [19].

\section{Etiology}

Rotator cuff disorders are the third most common musculoskeletal complaint and the number one cause of shoulder pain in patients over thirty [20]. According to a recent epidemiologic study, the incidence of rotator cuff pathology is $87 / 100,000$ person-years, with an increased prevalence in women compared to men ( 90 vs $83 / 100,000$ person-years) [21]. The highest incidence of rotator cuff disorders occurs between the ages of 55-59, and is more prevalent in lower socioeconomic groups [21]. Athletes who perform sports with overhead activity, such as swimming, tennis, pitching, lifting, volleyball, and gymnastics are at increased risk as well [12]. Rotator cuff disorders are also more common in the elderly, manual laborers, and those with occupations which require repetitive overhead motions [12]. Other risk factors for rotator cuff disorders include anatomic variants, scapular instability, and unstable or hypermobile glenohumeral joints $[12,22]$.

\section{Diagnosis}

Rotator cuff tendinitis can often be diagnosed with a detailed history and physical exam. The patient may recall a minor traumatic event or describe an insidious onset. Patients typically complain of shoulder pain with overhead motions; they may have difficulty performing daily activities such as putting on a shirt or brushing their hair [7]. Typically, the pain is described as antero-superior shoulder pain, often radiating to the lateral deltoid region [10]. Pain is aggravated with overhead activities, lying on the affected side, reaching, pushing, pulling, or lifting. Patients may complain of not being able to fall asleep or waking up during the night secondary to pain [13]. Once a sufficient history has been gathered, a physical exam is necessary to aid in the diagnosis.

The physical exam typically begins with inspection, looking for evidence of muscle atrophy. Keep in mind that patients will typically exhibit a lower shoulder position in the dominant shoulder compared to the non-dominant side. Patients with rotator cuff tendinitis frequently have a symmetric normal contour, although atrophy at the supraspinatus and infraspinatus fossa may be seen in extreme, chronic cases and result in prominence of the scapular spine. ${ }^{10}$ Palpation of the shoulder is performed, looking for tenderness over the affected muscles or focal subacromial tenderness at the lateral or posterolateral border of the acromion. Following palpation, range of motion should be assessed in the affected arm and compared to that of the contralateral upper extremity. A strength exam should be performed as well, asking the patient to move the extremity against resistance. Patients with rotator cuff tendinitis tend to experience pain with abduction greater than $90^{\circ}$ and/or with internal rotation.

Specific tests may be performed to assess the rotator cuff. A positive arc test is documented if a patient experiences pain between $60^{\circ}-120^{\circ}$ of active abduction and is best used in conjunction with the Neer test and Hawkins Kennedy test [23]. The painful arc test has a sensitivity and specificity of $53 \%$ and $76 \%$ respectively, though a positive test has a likelihood ratio of 3.7 , with a negative likelihood ratio of 0.36 , suggesting that is an effective test for diagnosing rotator cuff pathology $[24,25]$. The Neer test, used to assess impingement, is positive with a passive painful arc maneuver, with the examiner flexing the glenohumeral joint while preventing shoulder shrugging [23]. The sensitivity and specificity of the Neer test is $72 \%$ and $60 \%$, respectively [24]. The Hawkins Kennedy test, also used to assess impingement, is performed by the examiner stabilizing the shoulder with one hand while internally rotating the patient's arm with the elbow flexed at $90^{\circ}$ [23]. Its sensitivity and specificity is $79 \%$ and $59 \%$, respectively [24]. The Empty Can test, or Jobe Strength test, may be used to evaluate the supraspinatus, and has a positive and negative likelihood ratio of 2.08 and 0.31 , respectively [26]. It is performed by having the patient abduct the arm to $90^{\circ}$ with $30^{\circ}$ of forward flexion and full internal rotation of the shoulder. The physician then applies downward pressure to the arm while the patient resists. Pain without weakness indicates tendinopathy, while pain with weakness indicates that a rotator cuff tear may be present. The Gerber's Lift Off test, or Push Off test, assesses internal rotation and the function and strength of the subscapularis muscle. It is an effective test, with a positive and negative likelihood ratio of 8.50 and 0.27 , respectively [26]. The patient places the dorsal aspect of the hand against the back and is asked to elevate the hand away by pushing against resistance. ${ }^{23}$ Alternatively, the Belly Press test may be performed, by having the patient place both hands on the abdomen and push the elbows anteriorly [11]. Inability to elevate the hand away from the back (Lift Off Test) or maintain the elbows anterior to the trunk against gentle resistance (Belly Press Test) indicates possible subscapularis pathology.

A recent review by Hegedus et al. [24] examined clusters of shoulder examinations. A case study was used to illustrate the best available cluster of tests that could make a diagnosis of shoulder pathology. Clusters with a higher positive likelihood ratio (LR+) were more likely to rule in conditions and a lower negative likelihood ratio (LR-) would help rule out pathology. The best clusters from the current literature are described in the table 1.

Tests used to help distinguish rotator cuff tendinitis from a rotator cuff tear include the lag sign and lidocaine injection test. Lag is assessed when the patient is positioned at the end of a full range of passive motion and then instructed to maintain that position. A positive test is acknowledged if the extremity 


\begin{tabular}{|c|c|c|c|c|}
\hline Author(s) & Pathology & Test cluster & LR+ & LR- \\
\hline \multirow{3}{*}{ Litaker et al.,2000 } & \multirow{3}{*}{ Rotator cuff tear } & 1 Age $>65$ and & \multirow{3}{*}{9.84} & \multirow{3}{*}{0.54} \\
\hline & & 2 Weakness in external rotation & & \\
\hline & & 3 Night pain & & \\
\hline \multirow{4}{*}{ Park et al, 2005} & \multirow{4}{*}{ Rotator cuff tear (Full Thickness) } & 1 Age $\geq 60$ and & \multirow{4}{*}{28} & \multirow{4}{*}{0.09} \\
\hline & & $2+$ painful arc test & & \\
\hline & & $3+$ drop arm test & & \\
\hline & & $4+$ infraspinatus test & & \\
\hline \multirow{3}{*}{ Park et al, 2005} & \multirow{3}{*}{ Impingement } & $1+$ Hawkins-Kennedy and & \multirow{3}{*}{10.56} & \multirow{3}{*}{0.17} \\
\hline & & $2+$ painful arc test and & & \\
\hline & & $3+$ infraspinatus test & & \\
\hline \multirow{2}{*}{ Farber et al, 2006} & \multirow{2}{*}{ Anterior instability (traumatic) } & $1+$ apprehension test & \multirow{2}{*}{39.68} & \multirow{2}{*}{0.19} \\
\hline & & $2+$ relocation test & & \\
\hline \multirow{2}{*}{ Guanche \& Jones, 2003} & \multirow{2}{*}{ Labral tear } & $1+$ relocation test & \multirow{2}{*}{4.56} & \multirow{2}{*}{0.65} \\
\hline & & $2+$ active compression test & & \\
\hline \multirow{2}{*}{ Guanche \& Jones, 2003} & \multirow{2}{*}{ Labral tear } & $1+$ relocation test and & \multirow{2}{*}{5.43} & \multirow{2}{*}{0.67} \\
\hline & & $2+$ apprehension test & & \\
\hline
\end{tabular}

falls away, indicating a tear is more likely than tendinitis. ${ }^{10} \mathrm{An}$ externally rotated lag test has a positive likelihood ratio of 7.2, while an internally rotated lag test has a positive likelihood ratio of $5.6 .{ }^{25} \mathrm{~A}$ lidocaine injection test involves an injection of $1 \%$ lidocaine solution into the subacromial space via the lateral or posterolateral approach. In tendinitis, the pain will be relieved and the patient will exhibit normal muscle strength, whereas a rotator cuff tear will present with analgesia but continued weakness. The diagnostic accuracy of the physical exam is improved when different shoulder tests are used in combination with one another and in conjunction with specific historical findings. For instance, a positive physical exam in a patient who is over 39 years old, has a self-reported history of popping or clicking, and has a painful arc of motion is more likely to be rotator cuff tendinopathy[20].

Standard radiographs of the shoulder (true AP, axillary lateral, and scapular Y) are helpful adjuncts to the initial evaluation, as they provide information about overall bony anatomy, calcific tendinitis, glenohumeral joint osteoarthritis, or chronic signs of impingement, such as subacromial spurring or abnormalities at the greater tuberosity $[10,27]$. If symptoms still persist after 6 weeks of conservative therapy (ex: steroid injection and physical therapy), advanced imaging techniques may be utilized [28]. Ultrasound is able to evaluate the shoulder in motion, assess the contralateral shoulder in the same visit, is easy to perform, lowcost, and emits no radiation. Ultrasound has high sensitivity for tendinopathy, although it is more useful in diagnosing complete tears or determining tear extent [27]. A major downfall with ultrasound, however, is difficulty in seeing the entire rotator cuff. Ultrasound is also highly user dependent.

Magnetic Resonance Imaging (MRI) is the gold standard to visualize the rotator cuff tendons and evaluate for subacromial fluid, tendon inflammation, and rotator cuff tears (Figure 1). MRI helps determine the extent of rotator cuff tears, degree of muscular atrophy, breadth of fatty infiltration, and presence of biceps pathology [27]. It is used to rule out a tear if conservative therapy fails, to assess for a suspected tear, or to clarify an unknown diagnosis. MRI findings must be interpreted in the clinical context, as a recent observational study has shown that as high as $54 \%$ of asymptomatic patients over age 60 and $34 \%$ of all asymptomatic patients have rotator cuff tears [29]. Despite the differences in imaging modalities, a recent study by Fischer et al. [30] has shown a relatively accurate outcome in using ultrasound compared to the gold standard of MRI in assessing rotator cuff structure. The accuracy of ultrasound when using MRI as reference was $91.1 \%$ for the supraspinatus tendon, $84.4 \%$ for the infraspinatus tendon, $77.8 \%$ for the subscapularis tendon, and $86.7 \%$ for the long biceps tendon [30].

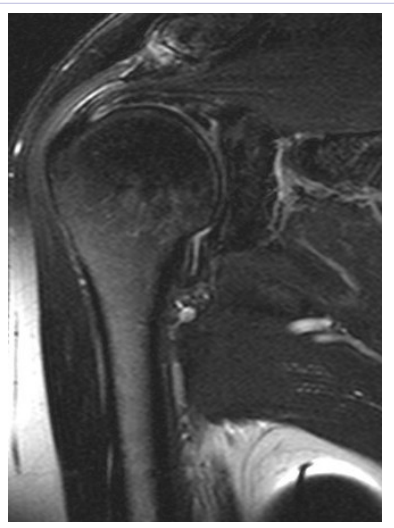

Figure 1: T2 coronal MRI demonstrating intermediate signal within the supraspinatus tendon and moderate tendon thickening consistent with tendinitis 


\section{Treatment}

Treatment principles for rotator cuff tendinitis include reducing pain and inflammation of tendons, preserving range of motion, and strengthening muscles. Although numerous nonoperative treatments are used in practice, little research has been done providing statistical significance of its efficacy. The initial treatment for rotator cuff tendinitis includes ice, rest, non-steroidal anti-inflammatory medications (NSAIDs), and corticosteroid injections [12]. Cryotherapy can decrease acute swelling and inflammation and provides some analgesia [8] Ice may be applied over the superolateral shoulder for 15 to 20 minutes every 4 to 6 hours. Rest is accomplished by avoiding overhead activity and other aggravating movements. NSAIDs, such as ibuprofen or naproxen, should be taken for 7 to 10 days for analgesia, though their anti-inflammatory effect in rotator cuff tendinitis is unclear [17]. There is currently no evidence that acetaminophen is less analgesic than NSAIDs. Opioids are reserved for moderate to severe pain; however, NSAIDs, acetaminophen, and tramadol should be tried first [31].

Continuing to work on shoulder range of motion is important to prevent increasing weakness and serious complications such as a frozen shoulder [32].Physical therapy focuses on range of motion improvement and maintenance, stretching, and strengthening exercises. Full range of motion should be attained prior to strengthening by first re-establishing passive arcs of motion [31]. Exercises include pendulum stretches, wand exercises (active assisted range of motion, using a pole), and wall walking (Figure 2). The goal of strengthening is to balance the rotator cuff muscles and scapular stabilizers [12]. Initial sets should be performed in non-impinging arcs, limited to waist-level internal and external rotation, followed by progression to active elevation and abduction in provocative zones at shoulder height and above [31]. Additionally, scapular stabilizers such as the trapezius, rhomboids, and serratus anterior should be addressed [33]. Eccentric exercise has been shown to stimulate healing by inducing a collagen synthesis response, as a differing amount and duration of torsional stress on the tendon provoke a diverse array of cellular reactions [34]. Although a single mechanism of tendon repair induced by eccentric exercises has not been proven, many theories exist, including creation of a larger load compared to concentric exercises, high-frequency oscillations in tendon force providing a stimulus for repair, increase in fibroblast activity, acceleration of collagen formation, increase in type I collagen, collagen organization/alignment (remodeling of the tendon) by muscular lengthening, an increase in the number of sarcomeres in series, a decrease in neovascularization, which is theorized to be associated with pain, and possible pain habituation [34].

A combined steroid and analgesic injection into the subacromial space is often the first line of treatment and may be trialed in conjunction with physical therapy. Ultrasound guidance to aid in placement has been shown to improve outcomes [35]. The benefit of steroids compared to NSAIDs is unclear. A recent meta-analysis of anti-inflammatory drugs in treating rotator cuff tendinopathy demonstrated that NSAIDs are effective at decreasing short-term pain, though they have no effect on

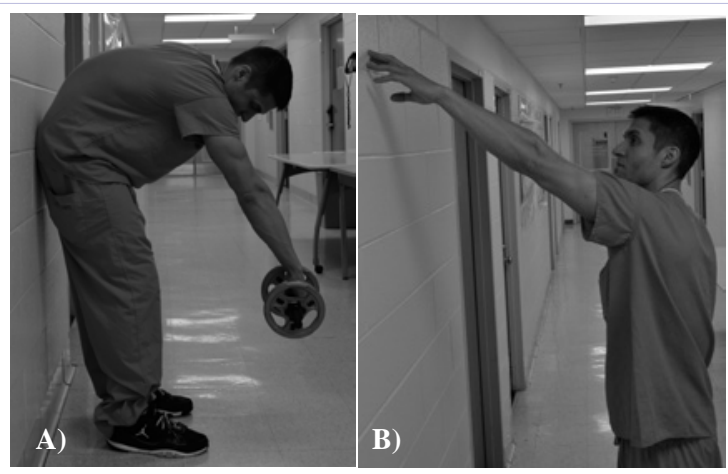

Figure 2: Example of stretching exercises using pendulum (A) and wall climbing (B) techniques.

function, and are equally as effective as corticosteroid injections $[36,37]$. Other studies have shown that steroids are more efficacious than NSAIDs and their higher doses provide more pain relief [18]. Similar discrepancies have been demonstrated with regard to length of efficacy; some studies show that corticosteroid injections can relieve shoulder pain caused by tendinitis for up to six weeks, with no benefit beyond six months, while others demonstrate that subacromial space injections can be effective for up to nine months $[6,18]$. Injections that do not provide relief do not warrant a second attempt, as it is not likely to help [33]. Predicting the outcome after a corticosteroid injection is difficult, with studies showing up to a $40 \%$ rate of failure [38]. A recent study performed by the Hospital for Special Surgery demonstrated that $1 / 3$ of patients failed conservative management and required surgery [38]. Previous studies have identified several predictors of a negative response to subacromial corticosteroid injections including duration of pain for over a year, degree of functional impairment on presentation, tear more than $1 \mathrm{~cm}$, female sex, dominant affected extremity, isolated infraspinatus injuries, and workers compensation claims $[39,40]$. However, a recent study by Contreras et al. [38] found no correlation of successful injections with age, hand dominance, or duration of symptoms. Potential side effects of these injections include injection site flares, increased blood sugars, increased tendon fragility, and periarticular calcification [41].

Multiple treatment modalities focused on improving underlying physiologic healing have been proposed in an effort to combat the decreased intrinsic capacity for tendon repair, which is touted as a common underlying etiology of rotator cuff tendinitis. Platelet-Rich Plasma (PRP) has been trialed in a variety of tendon injuries, including those in the knee, elbow, and shoulder. PRP is the cellular plasma component of whole blood containing a higher platelet concentration and various growth factors [42]. In vivo studies suggest that concentrated growth factors and biologically active substances within PRP are able to initiate the healing cascade within an area of tendon injury, thus having the potential to heal the muscle-tendon unit of the rotator cuff [43]. Early studies utilizing this treatment were promising, suggesting that ultrasound guided intralesional injections of PRP result in a safe, significantly sustained improvement in pain, function, and MRI outcomes in patients with refractory rotator 
cuff tendinitis [43]. However, a recent double blind randomized control study has shown that PRP had a similar efficacy as saline controls, suggesting that more studies may be needed to justify use of PRP as a primary non-surgical treatment modality [42]. Similarly, dry needling (percutaneous microtenotomy) has been attempted to transform chronic cuff injuries to more acute lesions with increased healing potential secondary to consequent bleeding and exposure to tissue growth factors. A recent trial comparing dry needling to PRP showed that both treatments had a positive effect, suggesting that the intrinsic growth factors may contribute to cuff healing [44].

Extracorporeal Shock-Wave Therapy (ESWT) is another treatment modality being investigated as a possible treatment for various shoulder pathology including rotator cuff injuries. While the mechanism remains unclear, it is hypothesized that healing occurs through fragmentation of calcification, neovascularization, and reduction of inflammation by decreasing chemokines, cytokines, and matrix metalloproteinases [45]. Many physical effects of ESWT depend on the energy delivered to a focal area, which is why compared to general ESWT, radial ESWT, which does not focus on a target zone, has shown to have less success $[45,46]$. This modality has also been trialed with adhesive capsulitis, calcific tendinitis, and other areas of tendonopathy such as the elbow and knee.

\section{Biceps Tendinitis}

\section{Anatomy}

The biceps brachii muscle is separated into the long and short heads. The short head originates on the coracoid process as part of the conjoined tendon with the coracobrachialis, while the long head originates on the superior labrum and supraglenoid tubercle. The long head of the biceps (LHB) is intra-articular as it passes over the humeral head (Figure 3) and becomes extra-articular as it enters the groove between the greater and lesser tuberosities. At this point, the tendon passes through a sling created by the superior glenohumeral ligament and the coracohumeral ligament, which acts as the biceps pulley. The tendon of the LHB extends along the anterior aspect of the humerus and converges with the tendon of the short head of the biceps, forming a common muscle belly. The distal biceps tendon inserts on the radial tuberosity and connects to the dorsal border of the ulna [47]. While muscular innervation arises from the musculocutaneous nerve, the tendon has sympathetic innervation that may contribute to the pathogenesis of shoulder pain [48].

\section{Pathology}

Biceps tendinitis refers to inflammation of the long head of the biceps tendon and the sheath within the intertubercular groove (Figure 4). The causes of biceps tendinitis can be separated into primary and secondary. Primary refers to inflammation in isolation, while secondary refers to the same inflammation when paired with other shoulder pathology, such as impingement syndrome or rotator cuff disease. Continued mechanical irritation leads to progressive inflammation and early tendinosis causing

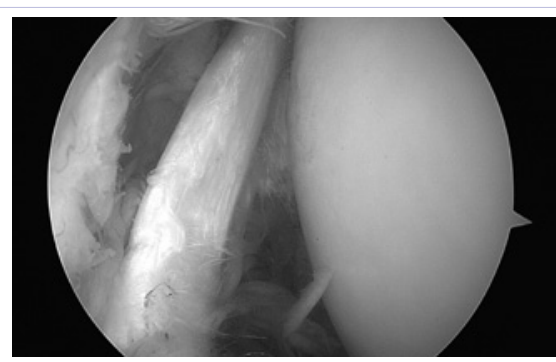

Figure 3: Long head biceps tendon with degenerative fraying visualized arthroscopically.

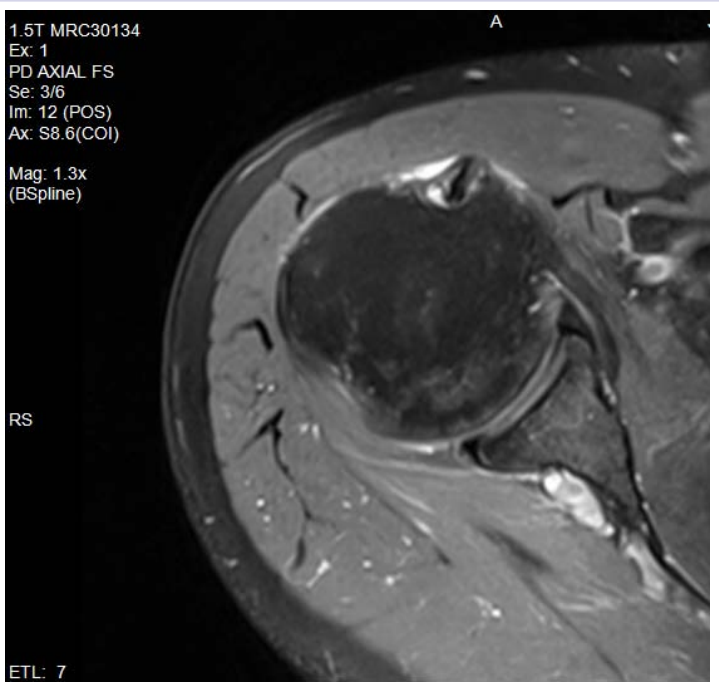

Figure 4: Proton-density axial MRI showing edema in the biceps tendon sheath and flattening of the long head biceps tendon within the intertubercular groove.

the LHB sheath to become thickened, fibrotic, and less vascular. Further inflammation may then lead to scar tissue and adhesions, which decreases the mobility of the LHB and predisposes it to rupture $[4,49]$. Alternatively, the tendon may demonstrate tendinosis, with degenerative changes rather than inflammation [50].

\section{Diagnosis}

Physical examination maneuvers and imaging can be used in combination to diagnose biceps tendinitis. Yergason's test is performed with the elbow flexed at $90^{\circ}$ and the forearm pronated, with the patient asked to supinate against resistance provided by the examiner [51]. A positive test is determined by pain elicited over the bicipital groove [52]. Speed's test is performed with the elbow extended and forearm supinated. A positive test is indicated by pain over the anterior aspect of the shoulder with resisted forward flexion [51]. Based on ultrasound findings, Yergason's test, Speed's test, and bicipital groove tenderness test have sensitivities of $32 \%, 63 \%$, and $57 \%$, respectively, and specificities of $78 \%, 58 \%$, and $72 \%$, respectively, in diagnosing biceps pathology [51]. To differentiate rotator cuff tendinitis from biceps tendinitis, a $10 \mathrm{~mL}$ injection of $1 \%$ lidocaine into the subacromial space can be performed. This should relieve the 
pain associated with rotator cuff tendinitis, but not that of biceps tendinitis [46].

\section{Treatment}

Initial non-operative treatment may consist of ice, antiinflammatories, rest, and activity modification. As pain improves, physical therapy may be initiated with a focus on range of motion [50,53]. Selective injection of corticosteroid into the subacromial space, glenohumeral joint, and biceps tendon sheath may be pursued to alleviate persistent pain. When injecting into the tendon sheath, direct injection into the tendon itself should be avoided, as this may predispose it to rupture $[4,54]$. Use of ultrasound may improve the accuracy of this injection. A recent review by Aly et al. [55] demonstrated that ultrasoundguided injections showed greater accuracy for all shoulder girdle injections, with the exception of the subacromial space, though subacromial space and biceps tendon sheath injections showed improved efficacy via improved function and reduction in pain. The accuracy of ultrasound versus a landmark-guided injection for shoulder joints were as follows: $93.6 \%$ vs $68.2 \%$ for the AC joint, $65 \%$ vs $70 \%$ for the subacromial space, $86.7 \%$ vs $26.7 \%$ for the biceps tendon sheath, and $92.5 \%$ vs $72.5 \%$ for the glenohumeral joint [55]. In throwing athletes with biceps tendinitis and other shoulder conditions, non-operative rehabilitation can be separated into rest, stretching, exercise, and progressive return to throwing. Stretching, including the hamstrings and low back, is initiated to regain range of motion and avoid stiffness and pain. When the shoulder is pain free, the patient may progress to exercise and strengthening followed by a graduated throwing regimen [56].

Although nonsurgical management of symptomatic biceps tendinitis is often recommended as the first-line treatment, the current literature is lacking regarding the efficacy and outcomes of this approach [4]. A Cochrane review examined 26 trials of physiotherapy interventions for shoulder pain. This review concluded that in "rotator cuff disease" there was evidence for mobilization and exercise and; that corticosteroid injections were superior to physiotherapy [57]. A study compared the use of joint injection with exercise, LHB sheath injection with exercise, heat and exercise, or analgesia in patients with 'periarthritis' of the shoulder. While nonspecific, the three groups that included exercise had better results than analgesia alone, but there was no significant difference between joint injection, LHB sheath injection, and heat groups with exercise [58]. A review of conservative treatment outcomes for tendinopathy at multiple common sites other than the biceps compared the use of an eccentric exercise programs to control groups including concentric exercise, stretching, splinting, massage, and ultrasound. The review suggests that eccentric exercise may decrease pain at twelve weeks of physical therapy versus twelve weeks of concentric exercise [59]. In cases where conservative treatment fails, patients may undergo surgical intervention consisting of either a biceps tenotomy or tenodesis depending on multiple factors including associated pathology, age, hand dominance, and activity level. [4]

\section{Adhesive Capsulitis}

\section{Anatomy}

Adhesive capsulitis is a common condition affecting the shoulder joint, resulting in a significant decrease in both passive and active ranges of motion. Other shoulder pathology can result in similar symptoms making the diagnosis difficult. The term "frozen shoulder," which Codman [60] originally described as linked to a tendinitis and inflammation of the subacromial bursa, is often incorrectly used as a synonym for adhesive capsulitis. Neviaser et al. [61,62] described pathologic specimens of adhesive capsulitis as a "chronic inflammatory process that produced a thickened and contracted capsule causing a synovial adherence to the humeral head". The mainstay of treatment is physical therapy and analgesics, however up to $10 \%$ of patients require some type of surgical intervention [63].

\section{Pathology}

Neviaser et al. [61,62] has contributed significantly to our current understanding of the progression of adhesive capsulitis. He described four stages through which the disease progresses. Stage 1 patients have nighttime pain near the deltoid insertion for less than three months. They can have some pain on physical exam with deep palpation or with passive stretch. Patients often lack external rotation while maintaining good rotator cuff strength. Arthroscopy and biopsy show minimal changes at this early stage, with mild inflammatory reaction and hypertrophic synovitis. Stage 2 shows a progression of the disease with increased pain, which is worse at night. Patients will also begin to report stiffness in more planes than just external rotation. At this stage, there is loss of range of motion even under anesthesia. Arthroscopy reveals mild loss of the axillary fold (Figure 5) and hypervascular synovitis. Biopsy confirms the hypervascular nature of the tissue along with subsynovial scar formation. In stage 3, patients have decreased pain, which is only found with the extremes of range of motion. The major complaint at this stage is stiffness. There is significant loss of motion in all directions on physical exam both passively and actively, which is not improved under anesthesia. Arthroscopy during this stage no longer reveals the intense hypervascular synovitis, but there is complete loss of the axillary fold. As would be expected, biopsy of tissue at this stage is similar to other fibrotic disorders with hypercellular collagenous tissue. In the final stage of the disease, there is very little pain, but profound stiffness. Arthroscopically, there are fully matured adhesions making identification of any intra-articular structures very difficult.

\section{Epidemiology}

Adhesive capsulitis affects $2-5 \%$ of population, peaks in the 40-60 year old age group, and has a higher incidence in females. The non-dominant hand is more often affected, however approximately $20-30 \%$ of patients will develop the disease in bilateral shoulders [64]. There is no known genetic predisposition. There are a significant number of medical conditions associated with the development of adhesive capsulitis including diabetes mellitus, autoimmune disease, stroke, myocardial infarction, 

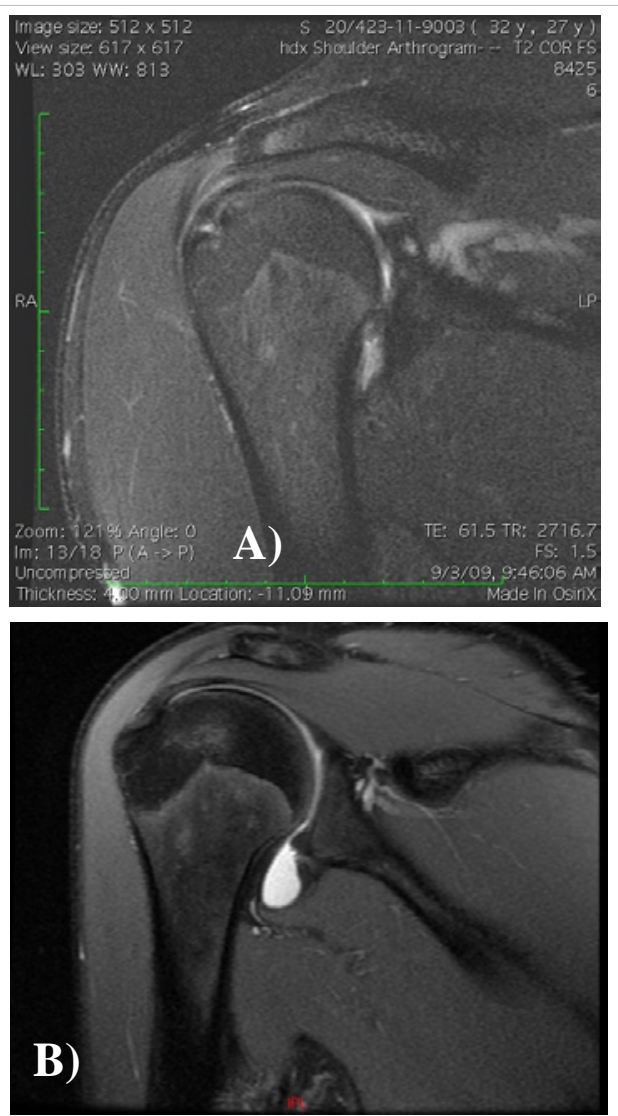

Figure 5: A: T2 coronal Magnetic Resonance Arthrogram (MRA) showing obliteration of the axillary pouch volume, B: T2 coronal MRA demonstrating normal axillary pouch volume. (Courtesy of Matthew Provencher, MD).

Parkinson's disease, Dupuytren's contractures, breast cancer, thyroid dysfunction, and sedentary lifestyles, possibly due to a predisposition for chronic inflammation $[63,65]$.

\section{Treatment}

Despite this condition being described for over 50 years, treatment guidelines often lack supportive literature. In fact, only corticosteroid injections have been given an " $A$ " grade as an intervention with strong level 1 supportive evidence for its use in adhesive capsulitis [66]. Most interventions currently rely on level III, level IV, or expert opinion. Some of the difficulty with designing treatment protocols is that patient-reported outcomes differ significantly from objectively measured outcomes [67]. Furthermore, adhesive capsulitis often resolves without any treatment $[68,69]$. Therefore, much of the treatment for adhesive capsulitis is targeted at providing symptomatic relief, rather than altering the course of the disease process.

Although good evidence supporting the efficacy of NSAIDs does not exist, they are still widely used as a first line therapy. NSAIDs provide some amount of pain relief, however there is no change in the disease process [70]. Intra-articular steroid injections have resulted in short-term improvement in several studies but demonstrate little difference in overall progression of adhesive capsulitis [71,72]. Furthermore, the efficacy of intraarticular steroid injections has not been studied in the various stages of the disease [71,72]. This theoretically could be an important consideration, as there seems to be significantly different histological and clinical findings in each of the four stages. Bulgen et al. [68] compared treatment with intraarticular steroid injections, physical therapy, ice, to no treatment and found no significant difference in any group at 6 months of follow-up. Improvement of pain is best achieved via subacromial injection, though there is no clear explanation as to why. This observation suggests that shoulder pain in patients with adhesive capsulitis may originate from the the subacromial bursa, by the spread of inflammatory mediators from glenohumeral region to thesubacromial bursa or vice versa through therotator cuff interval [73]. Despite limited evidence of treatment efficacy, corticosteroid injections remain one of the most common conservative treatments for adhesive capsulitis because several studies have suggested that they may decrease synovial inflammation, pain, and glenohumeral joint inflammation [41].

Hyalurons, which are glycosaminoglycans that form the backbone of proteoglycan aggregates in the extracellular matrix, are being used as a means of improving shoulder function for patients with osteoarthritis, rotator cuff tears, and adhesive capsulitis [41]. Several theories of the mechanism of action have been postulated and include decreased friction secondary to viscoelasticity, coating and protecting damaged cartilage, decreased inflammation, improved synovial fluid concentrations and synovium abnormalities. Lim et al. [41] has shown no significant difference in using hyaluronate versus corticosteroid injections in patients with adhesive capsulitis, making it a viable alternative.

The role of physical therapy in combating the disease process remains unclear. Most orthopedists prescribe physical therapy as a mainstay of treatment, despite a lack of strong evidence to support its use [74]. Some studies demonstrate that physical therapy may be more helpful early in the disease process [67]. Bulgen et al [68] and Carette et al [69] found no difference in objective shoulder scores following participation in a dedicated course of physical therapy using active range of motion and a variety of mobilization techniques over a period of six weeks to a year. Diercks et al. [74] showed that gentle physical therapy, using passive mobilization and stretching, provided better outcomes than aggressive manipulation, such as supportive therapy and exercise with pain limits. Despite physical therapy and pharmacologic treatment, surgical intervention consisting of an arthroscopic capsular release is required in $10 \%$ of cases [75]. Combining physical therapy with a less intense home exercise program has been shown to be less effective than corticosteroid injection alone for short term pain relief; it is unclear whether it would act as an effective adjunct [76].

Other non-operative treatment options being trialed for adhesive capsulitis include capsular distension, shockwave therapy, and electrotherapy. In theory, capsular distension functions by rupturing the stiffened joint capsule via hydropressure, thereby decreasing synovial inflammation and 
capsular fibrosis [77]. A normal shoulder joint volume capacity is $28-35 \mathrm{~mL}$ of injectable fluid, while a shoulder affected by adhesive capsulitis can contain as little as $5-10 \mathrm{~mL}$ of joint space volume [77]. Hydrodilation has been shown to be superior to manipulation of the shoulder under anesthesia as a means of increasing range of motion [77]. Shockwave therapy is used for adhesive capsulitis in a manner similar to that of rotator cuff tendinopathy. A recent study by Vahdatpour et al. [78] demonstrated that shockwave therapy has positive effects on treatment, quicker return to recovery, and improved quality of life. Electrotherapy attempts to increase energy into the body via electricity, sound, light, or thermal energy [79]. It is most often used as an adjunct to standard physical therapy. Low-Level Laser Therapy (LLLT) has been used to delivery light energy into the shoulder. Studies suggest that pain relief is achieved by reducing the level of pro-inflammatory cytokines and increasing antiinflammatory growth factors and cytokines [79]. It is unclear whether electrotherapy is more effective than placebo or if it is a useful adjunct to exercise [79].

\section{Conclusion}

Rotator cuff tendinitis, biceps tendinitis, and adhesive capsulitis are common disorders of the shoulder that result in significant patient morbidity. Non-operative management is generally focused on pain control while maintaining range of motion and strength of the shoulder girdle. Activity modification, oral anti-inflammatory medications, and steroid injections may reduce pain and soft tissue irritation secondary to inflammation. Improving passive and active range of motion of the shoulder may allow patients to resume activities of daily living with less discomfort and can prevent contracture of inflamed tissues. Strengthening of the shoulder girdle includes periscapular and cervical muscle exercises. Multiple repetitions with lower weights, eventually utilizing controlled eccentric exercises, allows for conditioning while minimizing further muscle injury. The efficacy of such modalities, however, varies depending on the etiology of shoulder dysfunction. Rotator cuff tendinitis has been shown to respond positively to non-operative treatment while adhesive capsulitis is not fully understood and there is little evidence clearly describing effective non-operative protocols. Additionally, multiple disorders of the shoulder may exist simultaneously, further complicating the clinical picture. Rotator cuff tendinitis, biceps tendinitis, and adhesive capsulitis frequently resolve completely with non-operative treatment; however, in the rare instances where pain and limited function persist, surgical intervention may be considered.

\section{Acknowledgement}

The investigation was performed at Drexel University College of Medicine.

\section{References}

1. Urwin M, Symmons D, Allison T, Brammah T, Busby H, Roxby M, et al. Estimating the Burden of Musculoskeletal Disorders in the Community: The Comparative Prevalence of Symptoms at Different Anatomical Sites, and the Relation to Social Deprivation. Ann Rheum Dis. 1998; 57(11):649-55.
2. Marinko LN, Chacko JM, Dalton D, Chacko CC. The Effectiveness of Therapeutic Exercise for Painful Shoulder Conditions: A MetaAnalysis. J Shoulder Elbow Surg. 2011; 20(8):1351-9. doi: 10.1016/j. jse.2011.05.013.

3. Littlewood C, Ashton J, Chance-Larsen K, May S, Sturrock B. Exercise for rotator cuff tendinopathy: a systematic review. Physiotherapy 2011. 98(2):101-9.

4. Nho SJ, Strauss EJ, Lenart BA, Provencher MT, Mazzocca AD, Verma $\mathrm{NN}$, et al. Long head of the biceps tendinopathy: diagnosis and management. J Am Acad Orthop Surg. 2010; 18(11):645-56.

5. Sarkar K, Uhthoff HK. Ultrastructure of the subacromial bursa in painful shoulder syndromes. Virchows Arch A Pathol Anat Histopathol. 1983; 400(2):107-17.

6. Andres BM, Murrell GA. Treatment of tendinopathy: what works, what does not, and what is on the horizon. Clin Orthop Relat Res. 2008; 466(7):1539-54.

7. Obaid H, Connell D. Cell therapy in tendon disorders: what is the current evidence? Am J Sports Med. 2012; 38(10):2123-32.

8. Khan KM, Cook JL, Bonar F, Harcourt P, Astrom M. Histopathology of common tendinopathies. update and implications for clinical management. Sports Med. 1999; 27(6):393-408.

9. Biberthaler P, Wiedemann E, Nerlich A, Kettler M, Mussack T, Deckelmann S, et al. Microcirculation associated with degenerative rotator cuff lesions. In vivo assessment with orthogonal polarization spectral imaging during arthroscopy of the shoulder. J Bone Joint Surg Am. 2003; 85-A(3):475-80.

10. Tashjian RZ, Keener JD, Yamaguchi K. Arthroscopic Treatment of Rotator Cuff Tears. In: Weisel SW, editor. Operative Techniques in Orthopaedic Surgery, Vol. 1. Philadelphia: Lippincott Williams \& Wilkins; 2012.

11. Thomson JC. Shoulder, Netter's Concise Orthopaedic Anatomy, 2nd ed. Philadelphia: Saunders; 2010.

12. Rosa TLD, Wang AW, Zheng MH. Tendinosis of the rotator cuff: a review. J Musculoskelet Res. 2001; 5: 143. doi: 10.1142/ S0218957701000544.

13.Van Der Windt, DA, Koes BW, De Jong BA, Bouter LM. Shoulder disorders in general practice: incidence, patient characteristics, and management. Ann Rheum Dis. 1995; 54(12):959-64.

14. Chakravarty K, Webley M. Shoulder joint movement and its relationship to disability in the elderly. J Rheumatol. 1993; 20(8): 1359-61.

15. Chansky HA, Iannotti JP. The vascularity of the rotator cuff. Clin Sports Med. 1991; 10(4): 807-22.

16. Apreleva M, Ozbaydar M, Fitzgibbons PG, Warner JJ. Rotator cuff tears: the effect of the reconstruction method on three-dimensional repair site area. Arthroscopy. 2002; 18(5): 519-26.

17. Factor D, Dale B. Current concepts of rotator cuff tendinopathy. Int J Sports Phys Ther. 2014; 9(2):274-88.

18. Rees JD, Wilson AM, Wolman RL. Current concepts in the management of tendon disorders. Rheumatology (Oxford). 2006; 45(5): 508-21.

19. Neviaser A, Andarawis-Puri N, Flatow E. Basic mechanisms of tendon fatigue damage. J Shoulder Elbow Surg. 2012; 21(2): 158-163. doi: 10.1016/j.jse.2011.11.014.

20. O'kane JW, Toresdahl BG. The evidenced-based shoulder evaluation. Curr Sports Med Rep. 2014; 13(5): 307-13. 
21. White JJ, Titchener AG, Fakis A, Tambe AA, Hubbard RB, Clark DI. An epidemiological study of rotator cuff pathology using The Health Improvement Network database. Bone Joint J. 2014; 96-B(3):350-3. doi: 10.1302/0301-620X.96B3.32336.

22. Mehta S, Gimbel JA, Soslowsky LJ. Etiologic and pathogenetic factors for rotator cuff tendinopathy. Clin Sports Med. 2003; 22(4):791-812.

23. Moen MH, De Vos RJ, Ellenbecker TS, Weir A. Clinical tests in shoulder examination: how to perform them. Br J Sports Med. 2010; 44(5):3705. doi: 10.1136/bjsm.2010.071928.

24. Hegedus EJ, Goode AP, Cook CE, Michener L, Myer CA, Myer DM, et al. Which physical examination tests provide clinicians with the most value when examining the shoulder? Update of a systematic review with meta-analysis of individual tests. Br J Sports Med. 2012; 46(14):964-78.

25. Hermans J, Luime JJ, Meuffels DE, Reijman M, Simel DL, Biermazeinstra SM. Does this patient with shoulder pain have rotator cuff disease?: The Rational Clinical Examination systematic review. JAMA. 2013; 310(8):837-47. doi: 10.1001/jama.2013.276187.

26. Lasbleiz S, Quintero N, Ea K, et al. Diagnostic value of clinical tests for degenerative rotator cuff disease in medical practice. Ann Phys Rehabil Med. 2014; 57(4):228-43. doi: 10.1016/j.rehab.2014.04.001.

27. Meislin RJ, Sperling JW, Stitik TP. Persistent shoulder pain: epidemiology, pathophysiology, and diagnosis. Am J Orthop (Belle Mead NJ). 2005; 34(12 Suppl):5-9.

28. Ardic F, Kahraman Y, Kacar M, Kahraman MC, Findikoglu G, Yorgancioglu ZR. Shoulder impingement syndrome: relationships between clinical, functional, and radiologic findings. Am J Phys Med Rehabil. 2006(1); 85: 53-60.

29. Sher JS, Uribe JW, Posada A, Murphy BJ, Zlatkin MB. Abnormal findings on magnetic resonance images of asymptomatic shoulders. J Bone Joint Surg Am.1995; 77(1): 10-15.

30. Fischer CA, Weber MA, Neubecker C, Bruckner T, Tanner M, Zeifang F. Ultrasound vs. MRI in the assessment of rotator cuff structure prior to shoulder arthroplasty. J Orthop. 2015; 12(1):23-30. doi: 10.1016/j. jor.2015.01.003

31. Iannotti JP, Kwon YW. Management of persistent shoulder pain: a treatment algorithm. Am J Orthop (Belle Mead NJ). 2005; 34(12 Suppl):16-23

32. Kuhn JE. Exercise in the treatment of rotator cuff impingement: a systematic review and a synthesized evidence-based rehabilitation protocol. J Shoulder Elbow Surg. 2009; 18(1): 138-60. doi: 10.1016/j. jse.2008.06.004.

33. Jonsson P, Wahlstrom P, Ohberg L, Alfredson H. (2006) Eccentric training in chronic painful impingement syndrome of the shoulder: results of a pilot study. Knee Surg Sports Traumatol Arthrosc. 2006; 14(1):76-81.

34. Camargo PR, Alburquerque-sendín F, Salvini TF. Eccentric training as a new approach for rotator cuff tendinopathy: Review and perspectives. World J Orthop. 2014; 5(5):634-44. doi: 10.5312/wjo.v5.i5.634.

35. Naredo E, Cabero F, Beneyto P, Cruz A, Mondejar B, Uson J, et al. A randomized comparative study of short term response to blind injection versus sonographic-guided injection of local corticosteroids in patients with painful shoulder. J Rheumatol. 2004; 31(2): 308-14.

36. Boudreault J, Desmeules F, Roy JS, Dionne C, Fremont P, Macdermid JC. The efficacy of oral non-steroidal anti-inflammatory drugs for rotator cuff tendinopathy: a systematic review and meta-analysis. J Rehabi

\section{Med. 2014; 46(4): 294-306. doi: 10.2340/16501977-1800.}

37. Koester MC, Dunn WR, Kuhn JE, Spindler KP. The efficacy of subacromial corticosteroid injection in the treatment of rotator cuff disease: a systematic review. J Am Acad Orthop Surg. 2007; 15(1):311.

38. Contreras F, Brown HC, Marx RG. Predictors of success of corticosteroid injection for the management of rotator cuff disease. HSS J. 2013; 9(1):2-5. doi: 10.1007/s11420-012-9316-6.

39. Bartolozzi A, Andreychik D, Ahmad S. Determinants of outcome in the treatment of rotator cuff disease. Clin Orthop Relat Res. 1994; (308):90-7.

40. Ekeberg OM, Bautz-holter E, Juel NG, Engebretsen K, Kvalheim S, Brox JI. Clinical, socio-demographic and radiological predictors of shortterm outcome in rotator cuff disease. BMC Musculoskelet Disord. 2010; 11:239. doi: 10.1186/1471-2474-11-239.

41. Lim TK, Koh KH, Shon MS, Lee SW, Park YE, Yoo JC. Intra-articular injection of hyaluronate versus corticosteroid in adhesive capsulitis. Orthopedics 37(10): e860-865. doi: 10.3928/01477447-2014092451.

42. Kesikburun S, Tan AK, Yilmaz B, Yasar E, Yazicioglu K. Plateletrich plasma injections in the treatment of chronic rotator cuff tendinopathy: a randomized controlled trial with 1-year follow-up. Am J Sports Med. 2013; 41: 2609-16.

43. Scarpone M, Rabago D, Snell E, Demeo P, Ruppert K, Pritchard P, et al. Effectiveness of platelet-rich plasma injection for rotator cuff tendinopathy: a prospective open-label study. Glob Adv Health Med. 2013; 2(2): 26-31. doi: 10.7453/gahmj.2012.054.

44. Rha DW, Park GY, Kim YK, Kim MT, Lee SC. Comparison of the therapeutic effects of ultrasound-guided platelet-rich plasma injection and dry needling in rotator cuff disease: a randomized controlled trial. Clin Rehabil. 2013; 27(2): 113-22. doi: 10.1177/0269215512448388.

45. Kolk A, Yang KG, Tamminga R, Van Der Hoeven H. Radial extracorporeal shock-wave therapy in patients with chronic rotator cuff tendinitis: a prospective randomised double-blind placebocontrolled multicentre trial. Bone Joint J. 95-B(11): 1521-6. doi: 10.1302/0301-620X.95B11.31879.

46. Speed CA. Fortnightly review: Corticosteroid injections in tendon lesions. BMJ. 2001; 323(7309):382-6.

47. Stevens K, Kwak A, Poplawski S. The biceps muscle from shoulder to elbow. Semin Musculoskelet Radiol. 2012; 16(4):296-315. doi: 10.1055/s-0032-1327004.

48. Alpantaki K, McLaughlin D, Karagogeos D, Hadjipavlou A, Kontakis G. Sympathetic and sensory neural elements in the tendon of the long head of the biceps. J Bone Joint Surg Am. 2005; 87(7): 1580-3.

49. Post M, Benca P. Primary tendinitis of the long head of the biceps. Clin Orthop Relat Res. 1989; (246):117-125.

50. Hsu SH, Miller SL, Curtis AS. Long head of biceps tendon pathology: management alternatives. Clin Sports Med. 2008; 27(4):747-62.

51. Chen HS, Lin SH, Hsu YH, Chen SC, Kang JH. A comparison of physical examinations with musculoskeletal ultrasound in the diagnosis of biceps long head tendinitis. Ultrasound Med Biol. 2011; 37(9):1392-8. doi: 10.1016/j.ultrasmedbio.2011.05.842.

52. Rm Y. Supination Sign. J Bone Joint Surg Am. 1931; 13(1):160.

53. Ahrens PM, Boileau P. The long head of biceps and associated tendinopathy. J Bone Joint Surg Br. 2007; 89(8): 1001-9. 
54. Ford LT, Debender J. Tendon rupture after local steroid injection. South Med J. 1979; 72(7):827-30.

55. Aly AR, Rajasekaran S, Ashworth N. Ultrasound-guided shoulder girdle injections are more accurate and more effective than landmarkguided injections: a systematic review and meta-analysis. Br J Sports Med. 2015; 49(16):1042-9. doi: 10.1136/bjsports-2014-093573.

56. Churgay CA. Diagnosis and treatment of biceps tendinitis and tendinosis. Am Fam Physician. 80(5): 470-6.

57.Green S, Buchbinder R, Hetrick S. Physiotherapy interventions for shoulder pain. Cochrane Database Syst Rev. 2003; (2):CD004258.

58. Lee M, Haq AM, Wright V, Longton EB. Periarthritis of the shoulder: a controlled trial of physiotherapy. Physiotherapy. 1973; 59(10):312-5.

59. Woodley BL, Newsham-West RJ, Baxter GD. Chronic tendinopathy: effectiveness of eccentric exercise. Br J Sports Med. 2007; 41(4): 18898; discussion 199.

60. Codman EA. The Shoulder: Rupture of the supraspinatus tendon and other lesions in or about the subacromial bursa. Boston: T. Todd Company; 1934.

61. Neviaser AS, Hannafin JA. Adhesive capsulitis: a review of current treatment. Am J Sports Med. 2010; 38(11): 2346-56. doi: 10.1177/0363546509348048.

62. Neviaser AS, Neviaser RJ. Adhesive capsulitis of the shoulder. J Am Acad Orthop Surg. 2011; 19(9):536-42.

63. Hand C, Clipsham K, Rees JL, Carr AJ. Long-term outcome of frozen shoulder. J Shoulder Elbow Surg. 2008; 17(2):231-6.

64. Binder AI, Bulgen DY, Hazlema BL, Roberts S. Frozen shoulder: a longterm prospective study. Ann Rheum Dis. 1984; 43(3):361-4.

65. Thomas SJ, Sarver JJ, Yannascoli SM, Tucker JJ, Kelly JD 4th, Ahima RS, et al. Effect of isolated hyperglycemia on native mechanical and biologic shoulder joint properties in a rat model. J Orthop Res. 2014; 32(11):1464-70. doi: 10.1002/jor.22695.

66. Kelley MJ, Shaffer MA, Kuhn JE, Michener LA, Seitz AL, Uhl TL, et al. Shoulder pain and mobility deficits: adhesive capsulitis. J Orthop Sports Phys Ther. 2013; 43(5):A1-A31. doi: 10.2519/jospt.2013.0302.

67. Griggs SM, Ahn A, Green A. Idiopathic adhesive capsulitis. A prospective functional outcome study of nonoperative treatment. J Bone Joint Surg Am. 2000; 82-A(10):1398-407.

68. Bulgen DY, Binder AI, Hazleman BL, Dutton J, Roberts S. Frozen shoulder: prospective clinical study with an evaluation of three treatment regimens. Ann Rheum Dis. 1984; 43(3):353-60.
69. Carette S, Moffet H, Tardif J, Bessette L, Morin F, Fremont P, et al. Intraarticular corticosteroids, supervised physiotherapy, or a combination of the two in the treatment of adhesive capsulitis of the shoulder: a placebo-controlled trial. Arthritis Rheum. 2003; 48(3): 829-38.

70.Van Der Windt DA, Van Der Heijden GJ, Scholten RJ, Koes BW, Bouter LM. (1995) The efficacy of non-steroidal anti-inflammatory drugs (nsaids) for shoulder complaints. A systematic review. J Clin Epidemiol. 1995; 48(5):691-704.

71. Arroll B, Goodyear-Smith F. Corticosteroid Injections for Painful Shoulder: A Meta-Analysis. Br J Gen Pract. 2005; 55(512):224-8.

72. Shah N, Lewis M. Shoulder adhesive capsulitis: systematic review of randomised trials using multiple corticosteroid injections. Br J Gen Pract. 2007; 57(541):662-7.

73. Kim SJ, Gee AO, Hwang JM, Kwon JY. Determination of steroid injection sites using lidocaine test in adhesive capsulitis: a prospective randomized clinical trial. J Clin Ultrasound. 2015; 43(6):353-60. doi: 10.1002/jcu.22201.

74. Diercks RL, Stevens M. Gentle thawing of the frozen shoulder: a prospective study of supervised neglect versus intensive physical therapy in seventy-seven patients with frozen shoulder syndrome followed up for two years. J Shoulder Elbow Surg. 2004; 13(5):499502.

75.Levine WN, Kashyap CP, Bak SF, Ahmad CS, Blaine TA, Bigliani LU. Nonoperative management of idiopathic adhesive capsulitis. J Shoulder Elbow Surg. 2007; 16(5):569-73.

76. Page MJ, Green S, Kramer S, Johnston RV, Mcbain B, Chau M, et al. Manual therapy and exercise for adhesive capsulitis (frozen shoulder). Cochrane Database Syst Rev. 2014; 8:Cd011275.

77. Bae JH, Park YS, Chang HJ, Kim MJ, Park KY, Jin SH, et al. Randomized controlled trial for efficacy of capsular distension for adhesive capsulitis: fluoroscopy-guided anterior versus ultrasonographyguided posterolateral approach. Ann Rehabil Med. 2014; 38(3):360-8. doi: $10.5535 / \mathrm{arm} .2014 .38 .3 .360$

78. Vahdatpour B, Taheri P, Zade AZ, Moradian S. Efficacy of extracorporeal shockwave therapy in frozen shoulder. Int J Prev Med. 2014; 5(7):87581.

79. Page MJ, Green S, Kramer S, Johnston RV, McBain B, Buchbinder R. Electrotherapy modalities for adhesive capsulitis (frozen shoulder). Cochrane Database Syst Rev. 2014; 10:Cd011324. 